\title{
Schielambulanz Graz damals und heute
}

\author{
Andrea Langmann · Susanne Lindner · Martina Christine Brandner · Heike Gaugl · Daniela Frühwirth-Kaspar · \\ Anna Maria Lienhart · Karin Steinmair
}

Jede meiner Vorlesungen für Studierende beginnt mit den Worten „Man kann seinen Beruf auch noch nach 35 Jahren mit Begeisterung ausüben, die Work-LifeBalance aufrecht erhalten und das Thema ,Familie und Beruf' leben“.

Wie dies unter den sich in der Medizin ständig ändernden Voraussetzungen und Anforderungen gelungen ist und wer dazu beigetragen hat, soll die folgende kurze Zusammenfassung der Entwicklung der Schielambulanz der Medizinischen Universität Graz (MUG) veranschaulichen.

Arbeitsbeginn in der Schielabteilung 7.30 Uhr: In den acht Untersuchungszimmern (vier für Orthoptik, vier für ärztlich-ophthalmologische Untersuchungen), am Anmeldeschalter und im Wartezimmer startet der Tag. Facharztzuweisungen mit Termin werden von Frau Petritsch und Herrn Lewonigg in den Schwerpunkten Schielen und Binokularstörungen, neurologische und mechanische Motilitätsstörungen, endokrine Orbitopathie, Kinderophthalmologie und Sehbehinderung bei Kindern (Dystrophien und kongenitale meist syndromale Anomalien) angemeldet, um orthoptisch und augenärztlich, einschließlich Skiaskopie, versorgt zu werden.

Unveränderter Schwerpunkt ist die Früherkennung und Behandlung der Amblyopie.

Die ursprüngliche routinemäßig durchgeführte Atropinskiaskopie machte einen mehrfachen Besuch, mühevolles, dreitägiges Tropfen zu Hause mit in der Folge im Allgemeinzustand reduzierten, lichtempfindlichen Kindern notwendig (auch die Mütter kamen

ao.Univ.-Prof. Dr. A. Langmann $(\bowtie) \cdot S$. Lindner ·

M. C. Brandner · H. Gaugl · D. Frühwirth-Kaspar ·

A. M. Lienhart · K. Steinmair

Universitätsaugenklinik, Medizinische Universität Graz,

Auenbruggerplatz 4, 8036 Graz, Österreich

andrea.langmann@klinikum-graz.at dabei häufig mit irrtümlich weiten Pupillen bei uns an). Ersetzt durch die heute übliche CyclopentolatSkiaskopie und in ausgesuchten Fällen den Photoscreener [1, 2] hat die Skiaskopie viel an Schrecken verloren. Klare Richtlinien zur Brillenverordnung [1], eine empfohlene Brillenadaptationszeit und Okklusionsstrategie sowie Möglichkeiten zur Verbesserung der Compliance erleichtern die Behandlung der Amblyopie. Die Zeiten der stationären Aufnahme zur Amblyopieschulung sind ebenso vorbei wie die Anwendung von Armmanschetten. Fragen nach der effizientesten Okklusionsdauer und der tatsächlichen Brillentragezeit werden derzeit in klinischen randomisierten prospektiven Multizenter-Studien [3], an denen auch wir teilnehmen, mittels „dose monitor“ ermittelt (Abb. 1). In der Literatur haben in den letzten Jahren neue Erkenntnisse zum Pathomechanismus der Amblyopie, im Speziellen der Einfluss auf das zweite, gesunde Auge und auf das Binokularsehen sowie daraus resultierende neue Behandlungsmöglichkeiten, einen großen Stellenwert eingenommen. So werden bessere Behandlungserfolge durch binokulares Training (Dichoptik) ebenso diskutiert wie die Möglichkeit, über perzeptuelles Lernen auch noch im Erwachsenenalter Einfluss auf die Schwachsichtigkeit nehmen zu können [4-7].

Nicht nur in Bezug auf die Amblyopie und deren Behandlung hat in der Strabologie die Berücksichtigung und Verbesserung der gesundheitsbezogenen Lebensqualität als Zielgröße an Bedeutung gewonnen [8, 9]. Möglicherweise auch aufgrund der steigenden Zahlen erwachsener PatientInnen mit Schiel- und/ oder Binokularproblemen [10, 11]. Waren Operationen ab dem 20. Lebensjahr vor 30 Jahren noch eine Seltenheit sind sie heute Standard geworden, wie wir auch in einer unserer Diplomarbeiten „Schieloperation im Erwachsenenalter - Entwicklung der letzten 10 Jahre, was erwartet uns in der Zukunft?“ 


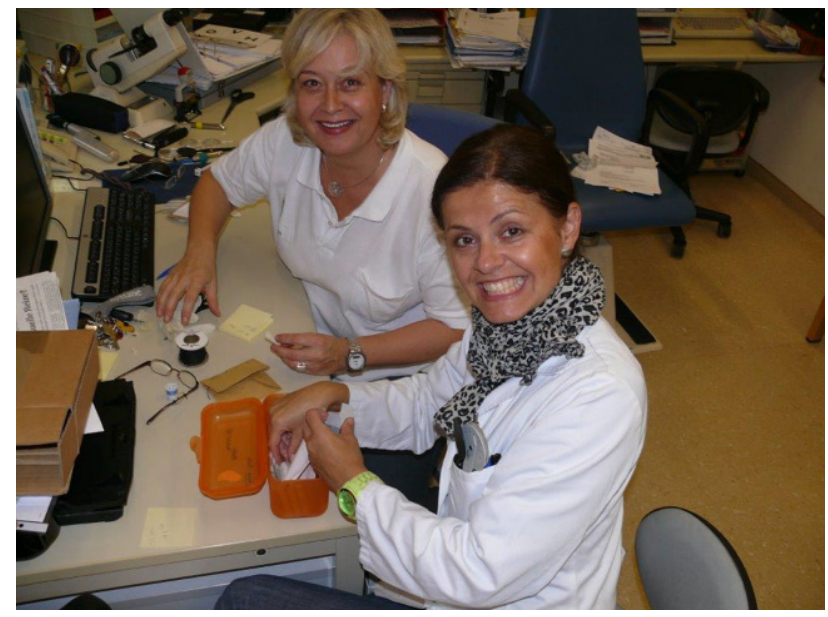

Abb. 1 Präparation „dose-monitor“

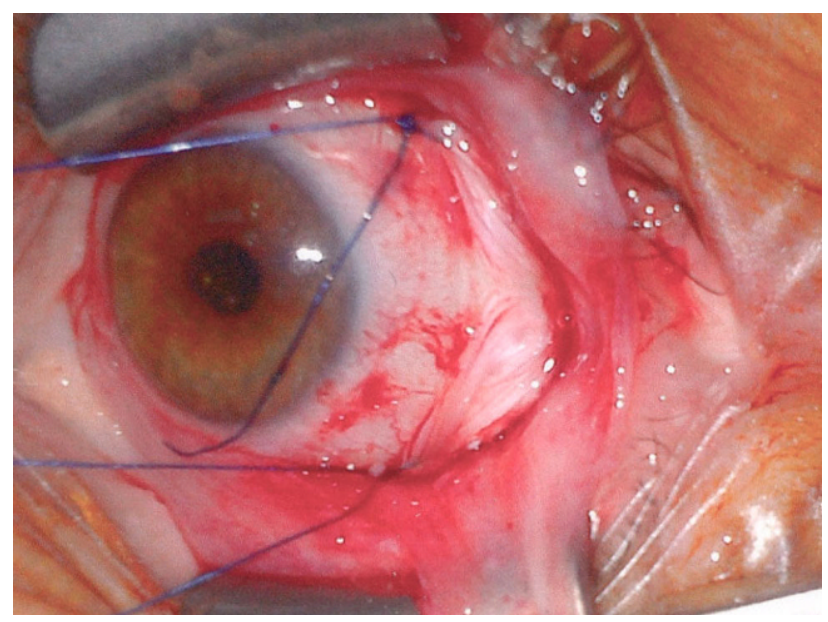

Abb. 2 Muskelteiltransposition nach Jensen

zeigen konnten. Die verbesserte Intensivmedizin und medizinische Betreuung neurologischer und neurochirurgischer PatientInnen, aber auch die guten Ergebnisse bei Reoperationen nach Schieloperation in der Kindheit sind hierfür ausschlaggebend. Komplexere Schieloperationen wie Transpositionen mit und ohne Botulinumtoxin (off-label in Österreich), Schlingenoperationen ([12]; Abb. 2), „hang back“ oder justierbare Fäden eröffneten eine breite Palette an Möglichkeiten zur Rehabilitation von Augenmuskelparesen mit Doppelbildern nach beispielsweise Schädelhirntraumata und Orbitaverletzungen. Neue Erkenntnisse zum Pathomechanismus der Motilitätsstörung bei endokriner Orbitopathie, maligner Myopie und beim „Sagging Eye Syndrome“ $[13,14]$ ermöglichen es Bewegungseinschränkungen zu beheben und ein doppelbildfreies Gebrauchsgesichtsfeld $\mathrm{zu}$ rekonstruieren. Manchmal ist es aber einfach nur der psychosoziale Druck auf Schielende in der heutigen Gesellschaft mit zunehmend kompetitiverem Arbeitsmarkt, der z. B. bei konsekutiver Divergenz eine neuerliche Stellungskorrektur notwendig macht. Im Gegensatz zu einem einstmals viertägigen sta- tionären Aufenthalt bei Schieloperation mit streng einzuhaltenden Besuchszeiten der Eltern, erfolgt die Operation heute altersunabhängig tageschirurgisch. Eine ausführliche präoperative Aufklärung mit genau standardisiertem Inhalt und Ablauf soll es der Patientin/dem Patienten in Abstimmung mit der Ärztin/dem Arzt ermöglichen die richtige Entscheidung für oder gegen die Operation zu treffen, Alternativen und Komplikationen zu überdenken und die dem möglichen Ergebnis gegenüber zu stellen.

Nicht nur die Operationsmethoden, das Nahtmaterial und die anästhesiologischen Möglichkeiten haben sich verbessert. Gerade im Hinblick auf die Diagnostik ist durch die heute differenzierte neuroorthoptische Abklärung zentraler Störungen der Okulomotorik häufig die Zuordnung von ophthalmologischen Beschwerden und Schwindel zur eventuell auslösenden zerebralen/zerebellären Läsion gelungen, selbst bei negativem bildgebenden Verfahren. Differentialdiagnostisch stehen PatientInnen mit endokriner Orbitopathie und Autoimmunmyositis im Mittelpunkt. Litten diese bis vor einigen Jahren unter den Nebenwirkungen der peroralen Kortisontherapie stehen heute mit der intravenösen Gabe von Kortison (Consensus statement der European Group on Graves' Orbitopathy EUGOGO) und neuen Biologika potente und weniger belastende Medikamente zur Verfügung. Auch die Schieloperation dieser dann mechanischen Bewegungseinschränkung lässt sich mit den heutigen Operationsmethoden gut dosieren und durchführen. Wesentlich an der Betreuung der PatientInnen sind mehr denn je eine internationale Vernetzung und die Kooperation mit den niedergelassenen KollegInnen, und anderen Disziplinen wie der Neurologie und Neurochirurgie, der Endokrinologie, Hals-NasenOhren-Klink oder der Kinderklinik. Nicht zu vergessen die KollegInnen der Psychologie/Psychosomatik, wenn Fragen zu Fusions- und Akkommodationsstörungen offen bleiben, Konversion von Aggravation differenziert werden muss, Akkommodationsspasmen vorliegen oder wenn die Differenzierung zur CVI (cerebrale visuelle Informationsverarbeitungsstörung) bei Aufmerksamkeits- und Lerndefiziten notwendig ist.

Von den Fortschritten in der Kinderophthalmologie profitierten besonders Sehbehinderte. Kunstlinsen und spezielle Kontaktlinsen ersetzen heute die Starbrille bei Aphakie, das kongenitale Glaukom ist dank verbesserter diagnostischer Mittel wie Tonopen oder I Care und verbesserter Operationstechniken leichter kontrollierbar und im Bereich der Netzhautdystrophien geben die Errungenschaften der Genetik und des „artificial vision“ Hoffnung auf Behandlungsmöglichkeiten. Trotz des immer niedrigeren Geburtsgewichtes unserer Frühgeborenen hat die Zahl an Frühgeburtenretinopathien abgenommen, was auf die bessere intensivmedizinische Betreuung aber auch auf bessere augenärztliche Behandlungsmethoden zurückgeführt werden kann. Der Diodenlaser hat die 
Cryotherapie abgelöst, und der Einsatz von intravitrealem anti VEGF [15] soll schwere Retinopathien verhindern, wenn auch viele Fragen zur Dosierung und zu systemischen Nebenwirkungen noch offen sind. Besondere Freude hat mir die Zusammenarbeit mit meinem Mann auf dem Gebiet der Retinoblastome bereitet - vorbei die Zeiten der primären Enukleation. Umso interessanter gestaltet sich nun die Betreuung erkrankter Tumorkinder, um nach bulbuserhaltender Therapie [16] durch Refraktionsausgleich, Amblyopiebehandlung und Schielkorrektur auch ein funktionell bestmögliches Ergebnis zu erhalten. Ausgehend von Graz hat vor über 30 Jahren in Kooperation mit dem Odilieninstitut die Sehfrühförderung und integrative Schulbetreuung Sehbehinderter begonnen, wodurch im wahren Sinn des Wortes „Mauern niedergerissen“ wurden.

Diese hohe Spezialisierung war sicherlich einer der Gründe weshalb ich mir diesen Fachbereich als Schwerpunkt gewählt habe. Noch heute bin ich dankbar für den großzügigen Umbau und die Erweiterung der damaligen Schielambulanz 1989 mit zahlreichen strukturellen und auch administrativen Veränderungen. In allen Vorgesetzten von Univ.-Prof. Hoffmann, Univ.-Prof. Hanselmayer („,.. ja, ja machst halt deine Ambulanz für sehbehinderte Kinder“), Univ.-Prof. Faschinger, Univ.-Prof. Faulborn bis zum heutigen Vorstand Univ.-Prof. Wedrich fand ich Unterstützer und Förderer. Dadurch war es mir immer wieder möglich das im Rahmen von Auslandsaufenthalten (Deutschland, Amerika, Niederlande) erworbene Wissen einzubringen. Wissen $\mathrm{zu}$ generieren und dieses auch praktisch, vor allem chirurgisch, umzusetzen sind allerdings zwei unterschiedliche Herausforderungen. Dazu braucht es verlässliche, hoch motivierte und kompetente KollegInnen. Glücklicherweise fand ich in Fr. OÄ. Dr. Susanne Lindner (Abb. 3a, b) eine treue, mich über Jahrzehnte begleitende Mitstreiterin. Mit Unterstützung der Mitglieder des CSA (besonders Frau Primaria Dr. Thaller-Antlanger, Herrn OMR. Dr. Todter, Frau Prof. Dr. Stangler-Zuschrott u. a.) war es einerseits möglich, als CSA-Vorsitzende und Mitglied des ÖOG Vorstandes innerhalb von Österreich Impulse zu setzen und andererseits bei der Akkreditierung der Fachhochschule „Studiengang Orthoptik" (für den Orthoptischen Dienst) mitzuwirken. Neben der Sicherung der Evidenz in der strabologisch/neuroorthoptischen Diagnostik und Therapie lag seit 2006 die Qualitätssicherung im Fokus unserer Bemühungen. Computerprogramme und eine elektronische Datenbank erlauben heute eine bessere Auswertung vor allem postoperativer Ergebnisse. Hier gilt der Dank unseren Orthoptistinnen Fr. Gaugl, Fr. Frühwirth-Kaspar, Fr. Lienhart und Fr. Steinmair, die im Rahmen einer jährlichen Klausur alle nach einem vorgegebenen Raster dokumentierten Ergebnisse präsentieren, sodass im Literaturvergleich auch Anpassungen vorgenommen werden können. Aus einer der hierbei geführten Diskussionen ergab sich auch
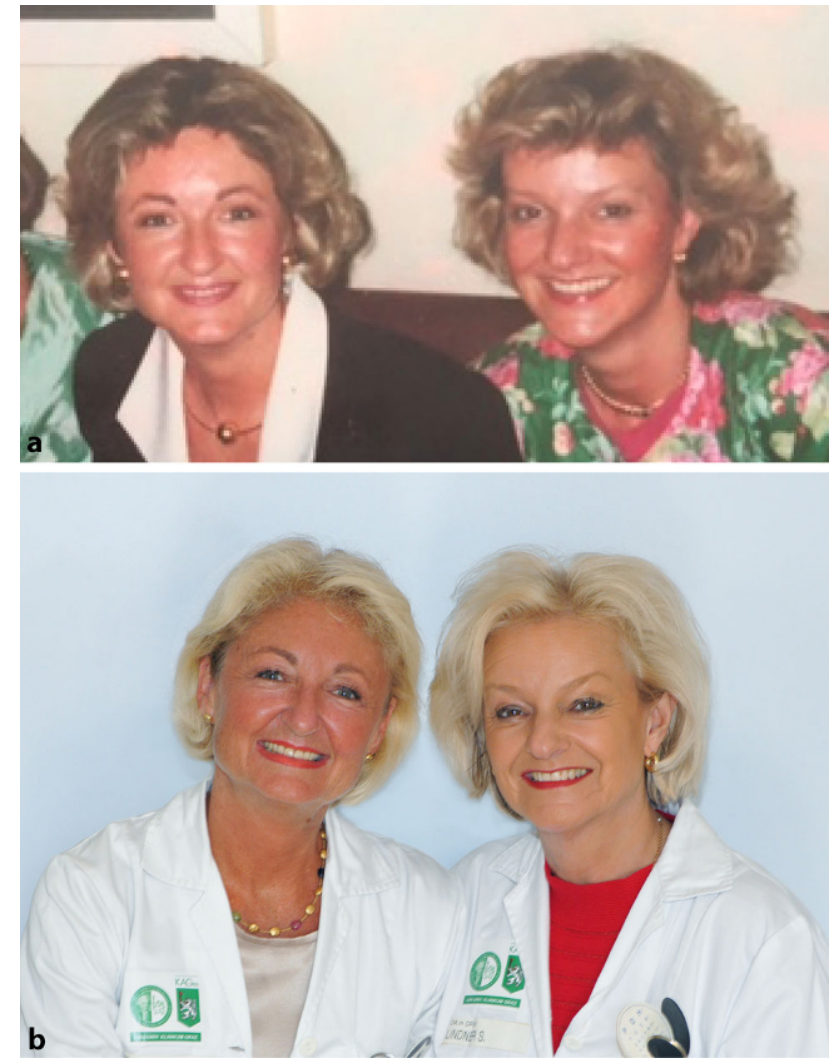

Abb. 3 a Damals und b heute

die Initiative „Schwindelambulanz“, eine Kooperation zwischen der Schielambulanz mit der Neurologie und der Hals-Nasen-Ohren-Klinik Graz.

Maximalen Einsatz benötigt es vom ganzen Team um den Anforderungen einer qualifizierten FacharztAusbildung gerecht zu werden. So wurde im Rahmen der Projektarbeit für das 2. Commited to Excellenz Assessment (C2E) der European Foundation for Quality Management (EFQM) der Univ.-Augenklinik 2010 ein strukturiertes Curriculum für die strabologische Ausbildung erstellt [17], das u. a. regelmäßige Orthoptik/logbook Stunden, Fallbesprechungen und praktische Übungen beinhaltet. 2015 wurde dieses an die neue Ärzte-Ausbildungsordnung angepasst und um ein Curriculum für die Schielchirurgie (,intermediate“ und „advanced“ Level nach ICO) erweitert. Dabei kann meine neue Kompetenz als Ausbildungsoberärztin der Univ.-Augenklinik Graz eingebracht werden. Das gesamte Curriculum zur Ausbildung zur AugenfachärztIn an der Augenklink Graz, angepasst an europäische (EBO) und weltweit aufgestellte Empfehlungen (ICO) lässt sich einschließlich der Lernziele u. a. auf unserer Homepage nachlesen [18]. Die individuelle Lernzielerreichung wird an der Univ.-Augenklinik Graz ebenso wie der Rotationsplan elektronisch dokumentiert.

Begleitende universitäre Funktionen zunächst als Gründerin und Vorsitzende des Arbeitskreises für Gleichbehandlung der neu ausgegliederten medi- 
zinischen Universität und später als Vizerektorin für Personal konnten gut genutzt werden, um die weiteren KollegInnen in Ihrer Entwicklung zu unterstützen und in Ihrer Laufbahn zu fördern. Eine eigene MentorInnenausbildung war dabei hilfreich. So befinden sich Fr. OÄ Brandner derzeit im laufenden Habilitationsverfahren und 2 KollegInnen im intermediate bzw. advanced level der strabologisch/ kinderophthalmologischen Ausbildung. Wir dürfen nicht vergessen, dass wir vielfach kleine PatientInnen behandeln und neue Erkenntnisse in der klinischen Forschung die Lebensqualität dieser PatientInnen über Jahrzehnte beeinflussen. Die zunehmende Anzahl an erwachsenen PatientInnen mit Binokularstörungen und Schielformen (angeboren oder erworben), die einer Korrektur bedürfen, weisen auf die Bedeutung des Bereichs in der Zukunft der Ophthalmologie hin: ein großer Spezialbereich mit vielen offenen Fragen für Forschungsthemen, deren Bearbeitung bei zunehmend kleinerer Anzahl an SpezialistInnen ohne zielgerichtete Ausbildung schwierig werden könnte.

\section{Zusammenfassung}

Bei aller Spezialisierung in der Medizin und Ophthalmologie muss der ganze Mensch, biopsychosozial im Mittelpunkt bleiben. Auch Binokularstörungen sind keine isoliert zu sehende Störung, sondern ein Begleitsymptom zahlreicher internistischer und neurologischer aber auch ophthalmologischer Erkrankungen wie Makulopathien, Glaukom, Netzhauterkrankungen, Sicca-Syndrom u. a. [14]. Die Dunkelzahl an unbehandelten PatientInnen, deren Beschwerden nicht richtig zugeordnet werden und die daher keine Therapie in Form von Prismenbrillen oder einfachen Schielkorrekturen erhalten, ist sehr hoch. Eine entsprechende Ausbildung und Erfüllung der im Rasterzeugnis eingeforderten Lernziele und eine gut überlegte Nachwuchsförderung im strabologisch/ neuroophthalmologischen Bereich würde auch einige standespolitische Fragen klären.

Ich bin stolz, an einer Augenklinik arbeiten zu dürfen, die 2017 die internationale Auszeichnung „Recognised for Excellence ${ }^{* * * *}$ (R4E 4 Stars) “ erreicht hat und sich nach Überschreiten von 400 Punkten im Assessment als exzellentes Unternehmen nach EFQM bezeichnen darf.

Dank und Respekt gilt meinem Team im Sekretariat, den Orthoptistinnen, meinen KollegInnen und im Besonderen meiner Familie.

Interessenkonflikt A. Langmann, S. Lindner, M. Brandner, H. Gaugl, D. Frühwirth-Kaspar, A. Lienhart und K. Steinmair geben an, dass kein Interessenkonflikt besteht.

\section{Literatur}

1. Ehrt O. Möglichkeiten und Grenzen des Amblyopiescreenings mit Autorefraktometern. Ophthalmologe. 2016;113:289-95.

2. Feldman S, Peterseim MM, Trivedi RH, Wilson ME, Cheeseman EW, Papa CE. Detecting high hyperopia: the plus lens test and the spot vision screener. J Pediatr Ophthalmol Strabismus. 2016;8:1-5.

3. Maconachie GD, Gottlob I. The challenges of amblyopia treatment. Biomed J.2015;38(6):510-6.

4. Eileen E. Birch1:amblyopiaand binocularvision. ProgRetin Eye Res. 2013;33:67-84.

5. Knox PJ, Simmers AJ, Gray LS, Cleary M. An exploratory study: prolonged periods of binocular stimulation can provide an effective treatment for childhood amblyopia. Invest OphthalmolVis Sci. 2012;53(2):817-24.

6. LiJ, Thompson B, Deng D, Chan LY, Yu M, Hess RF. Dichoptic training enables the adult amblyopic brain to learn curr. Biol.2013;23(8):R308-R9.

7. Li SL, Reynaud A, Hess RF, Wang YZ, Jost RM, Morale SE, De La Dao CAL, Stager D Jr, Birch EE. Dichoptic movie viewing treats childhood amblyopia. JAAPOS. 2015;19(5):401-5.

8. Gunton KB. Impact of strabismus surgery on health-related quality of life in adults. Curr Opin Ophthalmol. 2014;25(5):406-10.

9. Alpak G, Coskun E, Erbagci I, Bez Y, Okumus S, Oren B, Gurler B. Effects of corrective surgery on social phobia, psychological distress, disease-related disability and quality of life in adult strabismus patients. Br J Ophthalmol. 2014;98(7):876-9.

10. Stager D Jr. Adult Strabismus: it's never too late. J AAPOS. 2014;18(2):103-4.

11. Astle AT, Foulsham T, Foss AJ, McGraw PV. Is the frequency of adultstrabismus surgeryincreasing? Ophthalmic Physiol Opt. 2016;36(4):487-93.

12. Bizjak BS, Lindner S, Frühwirth-Kaspar D, Gaugl H, Lienhart A, Steinmair K, Langmann A. Beidseitige posttraumatische Abduzensparalyse. Spektrum Augenheilkd. 2017;31(1):14-8.

13. Chaudhuri Z, Demer JL. Sagging eye syndrome: connective tissue involution as a cause of horizontal and vertical strabismus in older patients. JAMA Ophthalmol. 2013;131(5):619-25.

14. Stangler Zuschrott E. Geriatric orthoptics and non-paretic diplopiain adults. New York: Nova SciencePublishers;2017. ISBN 10:1634852443.

15. Kandasamy Y, Hartley L, Rudd D, Smith R. The association between systemic vascular endothelial growth factor and retinopathy of prematurity in prematureinfants: a systematic review. Br J Ophthalmol. 2017;101(1):21-4. doi:10.1136/ bjophthalmol-2016-308828.

16. Langmann G, Langmann A, Sovinz P, et al. Vorschlag einer Vereinheitlichung der Retinoblastomtherapie in Österreich. Spektrum Augenheilkd.2004;18(1):32-7.

17. Tarmann L, Lindner S, Brandner M, Saliba S, Wedrich A, Foussek C, Klug U, Langmann G, Langmann A. European FoundationforQualityManagement(EFQM) at the division of strabology at the department of ophthalmology Graz. Spektrum Augenheilkd. 2012;26(3):165-71.

18. Langmann A, Langmann G, Rabensteiner D, Zenz H, Boldin, Hausberger, Langner-Wegscheider B, Mayer Ch, Pöschl EM, Wohlfart Ch, Palkovits St, Wedrich A. Ärzteausbildung neu 2015: Facharztausbildung neu im Sonderfach Augenheilkundeund Optometrie an der Universitäts-Augenklinik Graz. Spektrum Augenheilkd.2016;30(1):48-53. 